\title{
La culture humaniste littéraire en Master MEEF : un projet d'acculturation?
}

Catherine Dupuy

\section{(2) OpenEdition}

1 Journals

Édition électronique

URL : http://journals.openedition.org/trema/3346

DOI : 10.4000/trema.3346

ISSN : 2107-0997

Éditeur

Faculté d'Éducation de l'université de Montpellier

\section{Édition imprimée}

Date de publication : 1 mai 2015

Pagination : 112 - 121

ISSN : 1167-315X

\section{Référence électronique}

Catherine Dupuy, "La culture humaniste littéraire en Master MEEF : un projet d'acculturation ? »,

Tréma [En ligne], 43 | 2015, mis en ligne le 25 juin 2015, consulté le 21 avril 2019. URL : http:// journals.openedition.org/trema/3346 ; DOI : 10.4000/trema.3346

Ce document a été généré automatiquement le 21 avril 2019

Trema 


\title{
La culture humaniste littéraire en Master MEEF : un projet d'acculturation?
}

\author{
Catherine Dupuy
}

1 Les récentes réformes n'ont pas fait disparaitre la culture humaniste dans la formation universitaire professionnalisante des enseignants. Convoquée comme spécifique aux métiers de l'éducation, elle se retrouve dans les maquettes de Master MEEF (Métiers de l'Enseignement, de l'Éducation et de la Formation) sous forme d'Unité d'enseignement dans les mentions culture humaniste et culture scientifique. Nous examinons de plus près ici au travers de l'exemple de l'exercice d'écriture littéraire de l'épreuve de français du concours de professeurs des écoles les formes d'enseignement de la culture humaniste dispensées aux étudiants. Il s'agit d'envisager son enseignement universitaire moins en tant que discipline que d'une voie renouvelée d'apprentissage nécessaire à la professionnalisation du futur enseignant. À commencer par une approche plus vaste à la manière des «humanities» qui souligne l'importance du projet culturel dans la formation, des professeurs.

\section{Les contenus des enseignements de la culture humaniste en Master MEEF}

2 La maquette MEEF premier degré pour les étudiants destinés à devenir professeur des écoles contient dans le parcours de culture humaniste une unité d'enseignement intitulée «Acquérir les savoirs fondamentaux en français avec les objectifs suivants : l'acquisition des savoirs fondamentaux dans les domaines de la langue et la littérature; la réflexion sur la didactique du français; la maitrise de la méthodologie des exercices proposés au CRPE ».1

3 Tout d'abord, il est douteux que ces éléments de cadrage puissent valoriser la dimension historique des humanités classiques attachée à l'enseignement des Lettres. D'ailleurs, il 
n'est pas précisé que les contenus de ces enseignements fassent appel à des théories de références en langue et littérature. Ensuite, il apparait que la tendance est de faire étudier la langue française sous l'angle de sa maitrise avec «les excès de l'étude de la langue maternelle comme instrument de communication $»^{2}$ (Cibois ; 30).

Il semble alors que s'élabore au cœur même des enseignements MEEF la coupure entre une culture littéraire issue de la tradition scolaire des humanités et une culture technique du français centrée sur des formes de discours décontextualisées de la culture patrimoniale littéraire.

5 Afin de considérer la diffusion effective de la culture humaniste au-delà des libellés synthétiques des unités d'enseignement, on se propose d'examiner la méthodologie de lecture et d'écriture d'un des exercices proposé à l'épreuve de français du CRPE. Dans quelle mesure peut-elle être un vecteur de la diffusion de la culture humaniste littéraire auprès des étudiants?

\section{Culture humaniste et sujet lecteur littéraire}

6 Nous présentons un sujet d'entrainement de l'épreuve de français du concours de Professeurs des Écoles donné en formation. Il consiste à écrire une analyse ${ }^{3}$ littéraire à partir d'extraits de la Correspondance de Flaubert ${ }^{4}$ qui s'épanche auprès de Louise Colet sur son travail de création.

7 L'observation de ces documents compare l'écart entre les attendus du corrigé-type et les analyses rédigées par deux étudiants. L'exercice méthodologique; savoir lire et rédiger une analyse de texte littéraire contribue-t-il à développer une approche intéressée - dans le sens plein du terme - de la littérature? Gageons que l'exercice de l'analyse en dotant l'étudiant d'outils de lecture-écriture puisse cultiver « une attention » (Citton, 2014) ${ }^{5}$ à la littérature. Autrement dit, y a-t-il une place dans cet exercice d'épreuve de concours pour le sujet lecteur, notion phare de la recherche en didactique de la littérature? Nous livrons ci-dessous les différents supports utilisés dans la séquence d'enseignement.

9 Le début de la correspondance de Flaubert à Louise Colet du 4 avril 1854 se présente comme suit :

"J'ai été depuis vendredi dans un état affreux d'ennui et d'affaissement, résultat d'un passage dont je ne pouvais venir à bout. Il est, Dieu merci, passé depuis ce soir. Ce livre m'éreinte; j'y use le reste de ma jeunesse. Tant pis, il faut qu'il se fasse. La vocation, grotesque ou sublime, doit se suivre. Tu parles de ma quiétude. On n'a jamais parlé de rien de plus fantastique. Moi de la quiétude! Hélas! Non! Personne n'est plus troublé, tourmenté, agité, ravagé. Je ne passe pas deux jours ni deux heures de suite dans le même état. Je me ronge de projets, de désirs, de chimères, sans compter la grande et incessante chimère de l'Art."

Notons ce qu'une première lecture peut collecter pour une compréhension de l'extrait. D'un côté, des contenus d'histoire littéraire: le contexte d'écriture, les repérages biographiques, l'authenticité de cette lettre et la valeur de témoignage-documentaire sur le travail d'écrivain au XIXe siècle. D'un autre côté, une réflexion sur l'écriture littéraire ; en observant que le geste d'écriture de Flaubert est représentatif du travail universel de l'écrivain. Et en recueillant les confidences d'un grand écrivain qui "pense» son esthétique littéraire dans le genre épistolaire. 
11 Le corrigé type de l'analyse met en mots ce premier niveau de lecture comme on le voit ci-après :

"Pour G. Flaubert, le travail d'écriture constitue autant d'épreuves qu'il s'agit de subir : un douloureux parcours où l'auteur est assailli par des états émotionnels extrêmes d'ennui et d'affaissement. L'auteur annonce de ce fait la servitude du travail d'écriture qui l'oblige à se mettre à la tâche pendant la durée entière d'une saison. D'ailleurs, constate Flaubert, contraint de mobiliser ses forces et de faire des sacrifices, l'épuisement le guette. Le travail d'écriture implique un assujettissement aux contraintes imposées par le projet de création. »

Voici pour comparaison le texte écrit par l'étudiant 1:

"Pour Flaubert, les spécificités du travail d'écriture sont plutôt négatives, le travail d'écriture étant selon lui, en lien avec la douleur physique et le tourment perpétuel. En effet dans ces deux lettres, l'auteur utilise souvent le champ lexical de la douleur puisque pour lui le travail d'écriture "use le reste de sa jeunesse ». À travers les textes, on peut constater que selon l'auteur il s'agit d'un exercice lourd difficile physiquement; le but étant d'édifier quelque chose, d'avoir une production finale parfaite."

13 Cet extrait de rédaction se présente comme un paragraphe équivalent aux six lignes du corrigé type. Les deux productions débutent de la même manière en faisant référence au nom de Flaubert avec une énonciation à la troisième personne maintenue jusqu'à la fin. On relève d'autres structures en miroir : le corrigé-type utilise plusieurs fois des verbes à la voix active (4 occurrences) à la différence du texte de l'étudiant (2 occurrences). Il semble que le scripteur se soit auto normé dans sa formulation pour préserver une modalisation à la voix passive. Il a mis en évidence la maitrise linguistique de l'objectivité demandée par l'exercice. Ceci est confirmé par le nombre conséquent de tournures passives, support d'une énonciation impersonnelle.

Ci-après le texte de l'étudiant 2 complète la confrontation des documents.

"Ici, il convient de constater que tous les écrivains ont une aspiration à l'idéal lorsqu'ils écrivent une cuvre littéraire. Flaubert commence sa lettre en s'informant de l'état de santé de Louise Colet qui est souffrante, ce qui l'incite à parler de son propre état. En effet, Flaubert considère que l'écriture d'une œuvre est longue et pénible car chaque écrivain est passé par une détermination qui se transforme rapidement en acharnement afin de réaliser un écrit dont il pourra se satisfaire: "J'ai été depuis vendredi [...] venir à bout». Cet acharnement bascule ensuite dans le perfectionnisme et l'obsession d'un travail bien fait. Il faut que son écrit soit de qualité sinon il se doit de recommencer. Selon l'auteur de ces lettres, écrire est une vocation qui peut être perçue comme pathétique ou fascinante.»

15 L'énonciation ici se construit autour d'une progression linéaire servie par des tournures syntaxiques actives présentes jusqu'à la fin du paragraphe à propos des remarques de Flaubert sur le labeur de la création littéraire. Ainsi, on peut lire à la ligne 2. "Flaubert commence sa lettre en s'informant de l'état de santé de Louise Colet qui est souffrante, ce qui l'incite à parler de son propre état. " Auparavant, un premier énoncé à la voix unipersonnelle encadre le paragraphe. L'avant dernière séquence de l'extrait reprend une autre forme de dépersonnalisation de l'énonciation à l'aide d'un verbe à l'infinitif. On retrouve ici chez ce second scripteur comme chez le premier une rédaction désengagée d'analyse littéraire.

Néanmoins, on pourrait avancer que les écrits de ces deux étudiants donnent la preuve de «la rencontre avec l'œuvre $»^{6}$ (Chabanne et al., 2012) de Flaubert, médiatisée par trois instances le texte littéraire, le lecteur-formateur et le lecteur-étudiant. Il y a eu appropriation d'une énonciation distanciée, vecteur de lecture littéraire maitrisant les usages de la communication de l'écrit universitaire. Et on convoquerait Barthes énonçant que « La littérature, c'est ce qui s'enseigne, un point c'est tout » (Barthes, 1969- 1981). ${ }^{7}$ 
17 Pourtant, cela suppose que les étudiants aient aussi renoncé à une rencontre sensible. Même si on objectera que s'est installé un rapport au texte: rapport fonctionnel qui reformule les réflexions de Flaubert sur la création de son œuvre. C'est donc bien une manière de lire de type universitaire s'organisant autour du " plaisir du texte » (Barthes, 1973) ${ }^{8}$ comme premier rapport culturel qui affirme « la spécificité littéraire contre les sciences et les idéologues. » (Barthes, 1981) 9

Quelques traces, cependant de réactions émotionnelles apparaissent comme marques d'une lecture sensible au texte. Par exemple, un lexique évaluatif dans le texte de l'étudiant 2 reformule les difficultés de la création décrites par Flaubert avec les mots frappants comme

« acharnement et obsession ». S'exprime alors une forme subjective de discours critique littéraire portée par la métaphore médicale qui relève de la doxa culturelle assimilant folie et création littéraire.

Mais il s'avère que l'on ne puisse rien connaitre des « formes de vie » littéraires ${ }^{10}$ que les deux étudiants auraient investi à partir des connaissances enseignées dans le cadre de la séquence de culture humaniste. À savoir l'idéal de l'écriture de Flaubert, le sentiment de la langue et l'artisanat du métier d'écrivain. Il est vrai qu'il est difficile pour un scripteurétudiant de s'engager dans une expression plus ample : celle qui conduit à circuler entre une formulation d'opinions de sens commun littéraire et un travail d'intersubjectivité culturel et émotionnel avec un auteur.

20 Ainsi si la culture se définit "comme systèmes de valeurs de représentations et de comportements qui permettent à chaque groupe de s'identifier de se repérer et d'agir dans l'espace social environnant » (Cuche, 1996-2010, 50) ${ }^{11}$, on ne peut que s'interroger sur la pertinence du format contraint de l'exercice d'analyse à l'épreuve du concours.

21 Mais au-delà de cet exemple sur Flaubert, revenons sur les possibilités d'une formation spécifique à la culture humaniste littéraire.

\section{Retour sur une épreuve académique}

Comme dans d'autres concours de recrutement de la fonction publique ${ }^{12}$, la synthèse ${ }^{13}$ renvoie à une production d'écrit qui consiste à réduire les textes lus par le scripteur. Mais si la synthèse ${ }^{14}$ partage avec le résumé de textes l'exigence de se référer aux écrits donnés et d'en rendre compte aussi fidèlement que possible; elle demande par ailleurs une structuration formulée à partir d'une problématisation. Ce qui rend l'exercice paradoxal (Brassart, 1993) ${ }^{15}$. On demande de lire un corpus de textes pour les restituer en réduction dans une situation de communication mal définie. Le scripteur reformule «le dialogue » des idées et arguments entre les différents auteurs du corpus. Pour ce faire, il est contraint de gommer sa lecture dite subjective au profit d'une objectivité formelle dont on a pu repérer les modalités linguistiques dans les extraits des textes des étudiants sur Flaubert.

Convenons que nous sommes ici bien loin des avancées épistémologiques de la didactique de la littérature qui soulignent combien le lecteur est expert quand il circule aisément entre lecture subjective investie et distanciée (Dufays, 1996 ${ }^{16}$, Vibert, 2013 ${ }^{17}$ ).Mais également bien loin d'intégrer des ressources de la recherche en didactique du français comme les concepts de continuum scriptural ${ }^{18}$ (Dabène, 1987) de la réécriture $\mathrm{D}$. Bucheton (Bucheton, 2014) ${ }^{19}$ disponibles pour transmettre un modèle didactique 
d'écriture littéraire à des étudiants non spécialistes de la littérature que sont les futurs Professeurs des Écoles. Savoir écrire s'apprend tout au long d'un cursus scolaire avec des moyens rédactionnels adaptés aux situations diverses de communication écrite. À cet égard, le corpus de Flaubert peut être lu comme illustration du geste d'écriture qui se déploie selon un retour distancié et fusionnel sur le texte. La métaphore du monstre dévoré par sa propre écriture peut servir en formation à approcher l'isomorphie (toute relative, cependant) de l'artisanat exigeant de l'écriture littéraire et universitaire !

Ainsi, l'exemple de ce sujet de lecture-écriture littéraire montre que les apprentissages de l'exercice d'analyse dans les cours culture humaniste conduisent à proposer un enseignement formel qui ne demande en réalité aux étudiants que de réactiver leur méthodologie de la discipline Lettres du lycée.

On attendrait d'une formation des enseignants qu'elle reconfigure des éléments d'épistémologie universitaire de la culture humaniste.

Pour cela, trois dimensions pédagogiques devraient pouvoir servir de principes d'enseignement.

Celle que nous avons déjà évoquée caractérisant la culture fonctionnelle pour réussir une épreuve de concours qui associe un exercice de lecture-écriture.

À condition qu'elle soit prévue dans des dispositifs d'apprentissages ciblés (Masseron, 2004). ${ }^{20}$

Mais aussi celle qui acculture à un patrimoine lettré constitué de choix d'œuvres anciennes et contemporaines à diffuser auprès de professeurs des écoles non spécialistes de littérature. Sans oublier la visée de professionnalisation de la formation en culture humaniste dans sa dimension didactique. Il s'agit d'apprendre aux étudiants à didactiser des programmes de littérature à faire lire à de jeunes élèves.

Reste encore la question des frontières disciplinaires de la culture humaniste en formation d'enseignants. Nul doute que celle-ci n'a pas encore tenté d'expérimenter la réforme des

« humanities » déjà évoquées. Peut-on envisager en Master MEEF d'organiser l'ensemble des disciplines suivantes : l'interprétation des langues anciennes et modernes, l'éthique, l'histoire de l'art, la critique artistique et les théories des arts? Expérience de toute évidence encore impossible puisque nous rappelle Mongin, (Mongin, 2010, p132) ${ }^{21}$ «la géographie intellectuelle reste celle de la spécialisation des savoirs et de l'isolement des disciplines ".

\section{L'acculturation à la Culture Humaniste : spécificité des dispositifs en formation d'enseignants}

31 Permanence donc d'un enseignement universitaire qui forme "à » la littérature par le biais d'un exercice technique - qui initie cependant à la gymnastique savante de l'interprétation comme le faisait le latin des antiques humanités - mais qui renonce à former « par » la littérature comme le propose Martha Nussbaum.

Dans son ouvrage, les Émotions démocratiques. Comment former le citoyen du XXIe siècle ?22, cette spécialiste de la philosophie morale réhabilite les émotions comme faisant partie intégrante chez tout individu des raisonnements et interprétations qui médiatisent les objets du monde. À l'inverse, il apparait que se pérennise une tradition de la culture 
humaniste préoccupée par des apprentissages technicistes au détriment de la sollicitation d'expériences cognitives, culturelles et émotionnelles du lecteur-étudiant.

Peut-on espérer qu'une autre perception de la culture humaniste en Master MEEF - et ce au niveau des apprentissages visés dans l'exemple de l'analyse - s'impose, qui explicite pour mieux les traiter pédagogiquement les tensions d'un enseignement méthodologique formel de l'écriture articulées à des exigences rhétoriques et érudites requises pour un travail sur la littérature tout en sollicitant l'énonciation du sujet lecteur étudiant, futurenseignant? français en Master I, est selon nous sous estimé.

- Au niveau des tâches didactiques d'enseignement dévolues au formateur dans la maitrise du discours d'analyse littéraire; il s'agirait d'apporter des connaissances linguistiques et textuelles en « [faisant] observer des textes littéraires comme des réservoirs de solutions apportés à des problèmes d'écriture »(Petijean, 2003, p. 202).

- Au niveau de l'acculturation aux valeurs des textes littéraires, porter à la connaissance des étudiants des éclairages sur les travaux de sociologie culturelle (habitus, objets et capital culturels par exemple) complétés par des apports du domaine d'étude du contexte de l'œuvre dans les sciences du discours, le concept d'ethos (Maingueneau, 1993) ${ }^{24}$ inscrit dans la perspective de l'histoire culturelle (Chartier, 1998).25

- Corolaire du niveau précédent, l'acculturation aux valeurs éducatives au travers d'étude de groupement de textes de traités d'éducation de pédagogues en montrant que les thèmes retenus et les discours argumentatifs peuvent s'étudier pour en mesurer la valeur exemplaire. Ainsi peuvent se construire des apprentissages explicites qui amèneraient les étudiants à expliciter les valeurs mises en place dans les textes et à prendre position par rapport à leur propre système de croyances en éducation repérées dans les groupements de textes.

Tout ceci comme autant d'évolutions dans les contenus dans la maquette de Master M1 à décliner sous forme de gestes professionnels du formateur qui comme le préconise $\mathrm{D}$. Berthiaume $^{26}$ consiste à «choisir entre exhaustivité du contenu et profondeur de l'apprentissage ».

\section{Conclusion}

En bilan de cet article, signalons ce qui nous parait significatif dans la possibilité d'une culture humaniste spécifique en formation d'enseignants. Ce serait, et au risque de nous répéter, de concevoir un cadre de formation qui miserait sur les démarches pédagogiques d'acculturation qu'elle soit scientifique et/ou humaniste. Concrètement, envisager que se déploient dans les maquettes Master MEEF des parcours culture humaniste des 
enseignements de type «Éducation à » qui se formaliseraient comme suit : Éducation aux arts du langage.

Ainsi ce parcours offrirait l'occasion de contourner les usages technicistes universitaires des écrits sur la littérature que nous avons en partie pointés dans les documents sur l'analyse de Flaubert.

En bref, trouver les aménagements didactiques et pédagogiques afin que des futurs enseignants ne soient pas empêchés de lire «culturellement» les textes ${ }^{27}$. (Frier, Guernier, 2007).

Autant de moyens déployés au service de la constitution d'une nouvelle approche du métier d'enseignant qui sollicite l'étudiant en tant que sujet politique, psychologique et social à propos de ses capacités à éprouver l'expérience de la fiction littéraire.

Nul doute que le futur professeur puisse être acquis à ce que la culture littéraire soit un humanisme (professionnel).

\section{BIBLIOGRAPHIE}

Barthes, R., (1973.). Le plaisir du texte, Éd. du Seuil.

Barthes, R,. (1981). « Réflexions sur un manuel », l'enseignement de la littérature, De Boeck-Duculot, p.64.

Brassart, D.-G., (1993). « Remarques sur un exercice de lecture-écriture : la note de synthèse ou synthèse de documents ", Revue Pratiques, 79, 95-113.

Berthiaume, D., (2011). «Les pédagogies innovantes à l'université : Pourquoi ? Comment ? ", Congrès de l'ADBU, Vannes.

Bucheton, D. \& Alexandre, D., (2014). Refonder l'enseignement de l'écriture, Retz.

Chartier, R., (1999). Au bord de la falaise. L'histoire entre certitudes et inquiétude, Albin Michel.

Chabanne, J.-C., et al. (2012). La rencontre avec l'œuvre : éprouver, pratiquer, enseigner les arts et la culture, L'Harmattan.

Cibois, P., (2012). «L'avenir des Humanités » p.20 à 34, Refaire les Humanités, Esprit, décembre.

Citton, Y., (2014). Pour une écologie de l'attention, Seuil, La Couleur des idées.

Cuche, D., (2010). La notion de culture dans les sciences sociales, La Découverte, coll. « Grands Repères ".

Dabene, M., (1987). L'adulte et l'écriture, De Boeck.

Dufays, J.-L., Gemenne, L. et Ledur, D. , (1996). Pour une lecture littéraire 1. Approches historique et théorique, propositions pour la classe de français. Bruxelles, De Bœck -Duculot (Formation continuée).

Flaubert, G., (1974-76). Correspondance, Paris, Club de l'honnête homme, repris dans Gallica.

Frier, C. \& Guernier, M.-C., (2007). « Paroles de lecteurs : et si les usages scolaires empêchaient de pratiquer la lecture ? » Repères, 14 p. (halshs-00142575). 
Maingueneau, D., (1993). Eléments de linguistique pour le texte littéraire, Dunod.

Masseron, C., (2004). « Analyse critique de quelques dispositifs et activités d'écriture en DEUG », pp. 58-80, Les écrits universitaires, Pratiques, Numéro 121-122.

Mongin, O., (2010). « De la possibilité d'un avenir », De quoi l'avenir intellectuel sera-t-il fait ? enquêtes 1980, Le débat, Gallimard, pp.131-140.

Nussbaum, M., (2011). Les Émotions démocratiques. Comment former le citoyen du XXIe siècle ? trad.fr. par S. Chavel, Climats/Flammarion.

Petijean, A., 2003). « Histoire de l'écriture d'invention au lycée ». In Textes et valeurs, Pratiques, N -117/118, juin, pp. 190-202

Vibert, A., (2013). « Faire place au sujet lecteur en classe : quelles voies pour renouveler les approches de la lecture analytique au collège et au lycée? », http://eduscol.education.fr/lettres/ im_pdflettres/intervention-anne-vibert-lecture-vf-20-11-13.pdf

\section{ANNEXES}

\section{Annexes}

Extrait de dossier de demande d'habilitation à délivrer un Master : domaine SHS, DEG, STS, ALL. Mention Éducation et Formation, Montpellier 2, Montpellier 3 et Nîmes, publié en 2010.

«UE2 Culture humaniste, $60 \mathrm{~h}, 6$ ects, Semestre $1 \mathrm{C}$ et EC propose un enseignement systématique de la grammaire du français, de l'orthographe et du lexique. Il propose, en outre, des outils pour aborder la lecture littéraire, les genres littéraires, la littérature de jeunesse. [...].

\section{NOTES}

1. Concours de Recrutement de Professeurs des Écoles Cf. Annexes

2. Cibois, P., (Déc. 2012). « L'avenir des Humanités ", Refaire les Humanités, Esprit.

3. Notons que l'exercice de synthèse à l'épreuve de français se décline aussi comme une analyse de textes avec des citations à inclure dans le corps du texte rédigé. Nous adoptons donc pour l'exemple du sujet sur Flaubert le terme d'analyse.

4. Gustave Flaubert, Correspondance, (1974-76). Paris, Club de l'honnête homme, repris dans Gallica.

5. Citton,Y., (2014). Pour une écologie de l'attention, Seuil, La Couleur des idées.

6. Chabanne, J.-C. et al., (2014). La rencontre avec l'œuvre : éprouver, pratiquer, enseigner les arts et la culture, L'Harmattan.

7. Barthes, R., (1971), «Réflexions sur un manuel », L'enseignement de la littérature, dir. S. Doubrovsky et T. Todorov, Librairie Plon, réédition partielle, De Boeck-Duculot, 1981, p.64.

8. Barthes, R., (1973). Le plaisir du texte, Éd. du Seuil.

9. Barthes, R., (1971), "Réflexions sur un manuel ", L'enseignement de la littérature, dir. S. Doubrovsky et T. Todorov, Librairie Plon, réédition partielle, De Boeck-Duculot, 1981

10. En référence aux formes de vie de Wittgenstein, Recherches philosophiques, (2005), trad. Gallimard. 
11. Denys, D., (2010). La notion de culture dans les sciences sociales, La Découverte, coll. «Grands Repères ».

12. L'École Nationale d'Administration fondée en 1945 et réformée en 1971, institue la note de synthèse comme un outil de sélection pour recruter des élèves qui sachent dégager des éléments essentiels d'un dossier qui convoquent des savoirs juridiques économiques et politiques.

13. Depuis le 26 mai 2005 (texte actuel de référence), l'épreuve se présente comme suit:» à partir d'un dossier composé de textes et de documents relatifs à l'acquisition et à l'enseignement de la langue française, le candidat: - effectue une synthèse à partir d'une question relative au dossier ; - traite un thème ayant trait à la grammaire ; - répond à une question complémentaire sur la mise en situation d'enseignement d'une ou plusieurs notions abordées dans le dossier?».

14. Comme mentionné plus haut et pour simplifier le propos les dénominations de l'exercice de synthèse et d'analyse recouvrent un même exercice.

15. Brassart, D.-G., (1993). Remarques sur un exercice de lecture-écriture : la note de synthèse ou synthèse de documents. Revue Pratiques, 79, 95-113.

16. Dufays, J.-L., Gemenne, L. et Ledur, D. (1996). Pour une lecture littéraire1. Approches historique et théorique, propositions pour la classe de français. Bruxelles, De Bœck - Duculot (Formation continuée).

17. Vibert, A. http://eduscol.education.fr/lettres/im_pdflettres/intervention-anne-vibertlecture-vf-20-11-13.pdf

18. Dabene, M., (1987). L'adulte et l'écriture, De Boeck.

19. Bucheton, D. \& Alexandre, D., (2014). Refonder l'enseignement de l'écriture, Retz.

20. Masseron, C., (2004). "Analyse critique de quelques dispositifs et activités d'écriture en DEUG », pp. 58-80, Les écrits universitaires, Pratiques, Numéro 121-122.

21. Mongin, O., (2010). p132.

22. Nussbaum, M., (2011). Les Émotion démocratiques. Comment former le citoyen du XXIe siècle ? trad. fr. par S. Chavel, Climats/Flammarion.

23. Berthiaume, D. (2011). Les pédagogies innovantes à l'université : Pourquoi ? Comment? Congrès de l'ADBU, Vannes.

24. Maingueneau, D., (1993). Éléments de linguistique pour le texte littéraire, Dunod.

25. Chartier, R., (1999). Au bord de la falaise. L'histoire entre certitudes et inquiétude, Albin Michel, Paris.

26. Berthiaume, D. (2011). Les pédagogies innovantes à l'université : Pourquoi ? Comment? Congrès de l'ADBU, Vannes.

27. Nous paraphrasons ici une partie du titre de l'article suivant Catherine Frier, Guernier Marie-Cécile. «Paroles de lecteurs : et si les usages scolaires empêchaient de pratiquer la lecture ?» (2007), Repères, 14 p. halshs-00142575.

\section{RÉSUMÉS}

Quel sens redonner aujourd'hui à la culture humaniste? Si cette question est régulièrement évoquée dans les débats sur l'Éducation, elle l'est moins à propos de la formation initiale des enseignants. Les masters MEEF (Métiers de l'Enseignement, de l'Éducation et de la Formation) dispensent des enseignements qui privilégient des textes et des auteurs de la culture héritée Humaniste et Scientifique. Pourtant, reste à savoir quel bilan tirer de la réelle diffusion de la 
culture littéraire auprès d'étudiants, futurs-enseignants. Pour cela, on s'attardera sur le contexte institutionnel de la formation universitaire des enseignants qui fait en partie obstacle à l'enseignement effectif de la culture humaniste littéraire. Ensuite, l'analyse d'écrits d'étudiants à propos de leur lecture de Flaubert permettra d'envisager quelques propositions pour une acculturation des Humanités littéraires nécessaire à la formation professionnelle des enseignants.

Which feel falling in the humanist culture today? If this question is regularly evoked in the debates on the Education, it is less about the initial formation of the teachers. Master's degrees MEEF by the Education and by the Formation dispense teachings which favor texts and authors of the inherited culture Humanist and Scientist. Nevertheless, it remains to be seen which balance assessment to pull of the real distribution of the literary culture with student, future-teaching. For that purpose, we shall linger over the institutional context of the university education of the teachers which puts partially obstacle to the actual education of the literary humanist culture. Then, the analysis of students' papers about their reading.

\section{INDEX}

Mots-clés : acculturation aux humanités, culture humaniste littéraire, formation universitaire professionnelle des enseignants

Keywords : acculturation in the humanities, literary humanist culture, professional university education of the teachers

\section{AUTEUR}

\section{CATHERINE DUPUY}

Faculté d'Éducation - Université de Montpellier, ESPE-LR, LIRDEF/ALFA 